\title{
PharmaNews
}

Oncol Res Treat 39 | 9 | 16

\section{Zulassungserweiterung Ibrutinib}

\section{Neue Erstlinientherapie für CLL-Patienten}

Mit der Zulassungserweiterung zur Erstlinientherapie können nun Patienten mit chronischer lymphatischer Leukämie (CLL) über alle Therapielinien hinweg von der Behandlung mit Ibrutinib (Imbruvica ${ }^{\circledR}$, von Janssen) als Einzelsubstanz profitieren. Dies bedeutet einen weiteren wichtigen Schritt zur Verbesserung in der zielgerichteten Krebstherapie. Der Bruton-Tyrosinkinase (BTK)-Inhibitor Ibrutinib erhielt am 26. Mai 2016 die Zulassung zur Erstlinienbehandlung von bisher unbehandelten CLL-Patienten [1]. ${ }^{*}$ Bereits seit Herbst 2014 ist Ibrutinib zur Behandlung von Patienten mit rezidivierter/refraktärer (rez./ref.) $C L L$ und zur Erstlinienbehandlung von CLL-Patienten mit 17p-Deletion oder TP53-Mutation und für Patienten, die für eine Chemoimmuntherapie nicht geeignet sind, zugelassen.

\section{Erstlinientherapie: Ibrutinib}

Chlorambucil deutlich überlegen

Grundlage der Zulassungserweiterung war die randomisierte, multizentrische, zweiarmige Phase-III-Vergleichsstudie RESONATE-2. Diese untersuchte erstmals die Wirksamkeit und Verträglichkeit von Ibrutinib als Einzelsubstanz gegenüber Chlorambucil in der Erstlinientherapie bei 269 therapienaiven, behandlungsbedürftigen, älteren CLL-Patienten [2]. Für Patienten mit unbehandelter CLL belegten die Ergebnisse der RESONATE-2-Studie die überlegene Wirksamkeit des oral ein- genommenen BTK-Inhibitors $(\mathrm{n}=136)$ gegenüber der Chlorambucil-Therapie $(n=133)$ sowohl im progressionsfreien Überleben (PFS; Independent Review Committee (IRC)-Bewertung; primärer Endpunkt) als auch in den sekundären Endpunkten wie Gesamtüberleben (OS), hämatologische Verbesserungsrate, Gesamtansprechrate (ORR) und Sicherheit. Die Studie von Burger et al. [2] zeigte, dass Ibrutinib nach einem medianen Follow-up von 18,4 Monaten das Risiko auf Krankheitsprogression oder Tod der Patienten signifikant um $84 \%$ gegenüber Chlorambucil senkte $(\mathrm{p}<0,001)$. Außerdem lebten Patienten unter Ibrutinib signifikant länger ohne Krankheitsprogression als unter Chlorambucil. Ibrutinib zeigte sich als signifikant überlegen sowohl im primären als auch in den sekundären Endpunkten, obwohl Patienten des Chlorambucil-Arms bei Krankheitsprogression die Möglichkeit des Crossovers in den Ibrutinib-Arm gegeben war. Nach 1,5 Jahren waren 90\% der therapienaiven CLL-Patienten im Ibrutinib-Arm progressionsfrei versus 52\% der Patienten unter Chlorambucil (Abb. 1). Bei Patienten im Chlorambucil-Arm dauerte es im Mittel 18,9 Monate bis zur Krankheitsprogression. Im Vergleich dazu wurde das mediane PFS im Ibrutinib-Arm noch nicht erreicht (prim. Endpunkt; p < 0,001; Hazard Ratio (HR) 0,16; 95\%-Konfidenzintervall (KI): 0,09-0,28). Die beobachtete OS-Rate der mit Ibrutinib therapierten Patienten war beeindruckend. Nach

2 Jahren waren 98\% der CLL-Patienten im Ibrutinib-Arm am Leben, unter Chlorambucil waren es nur $85 \%$ (sek. Endpunkt; p $<0,001$; HR: 0,16; 95\%-KI: 0,05-0,56; Abb. 2) [2]. Die deutliche Verbesserung des OS unter Ibrutinib bestätigt die Daten des 3-jährigen Follow-ups einer Phase-II-Studie, in dem 31 symptomatische, unbehandelte Patienten $\geq 65$ Jahre eine 30-Monats-OS Rate von 97\% (95\%-KI: 78 99,5\%) und 101 vorbehandelte CLL-Patienten ein 30-Monats-OS von 79\% (95\%-KI: 69-86\%) aufwiesen [3].

Die therapienaiven CLL-Patienten sprachen mehr als doppelt so häufig auf eine Therapie mit Ibrutinib an als auf Chlorambucil (86\% vs. 35\%; p < 0,001; sek. Endpunkt) [2]. Die gute Ansprechrate bestätigte ebenfalls die bereits veröffentlichten ORR-Daten (89\%) der 3-jährigen Langzeitbeobachtung [3].

\section{Ibrutinib: Verbesserte Knochenmarksfunktion}

Als Verbesserung der Knochenmarksfunktion definierten die Studienleiter einen kontinuierlichen anhaltenden Anstieg des Hämoglobinwertes oder der Thrombozytenzahl über mindestens 56 Tage $^{\star *}$. Die Messungen zeigten eine signifikant verbesserte Knochenmarksfunktion bei Patienten unter Ibrutinib im Vergleich zur Kontrolltherapie. Der Anstieg des Hämoglobinwertes betrug im Ibrutinib-Arm 84\% versus $45 \%$ im Chlorambucil-Arm ( $p<0,001$; sek. Endpunkt) [2]. Auch die anhaltende Verbersserung der Thrombozytenanzahl war signifikant ausgeprägter unter Ibrutinib (77\%) als im Vergleichsarm $(43 \% ; \mathrm{p}=0,005$; sek.

* Ibrutinib ist als Einzelsubstanz zur Behandlung erwachsener Patienten mit nicht vorbehandelter chronischer lymphatischer Leukämie (CLL) zugelassen. Ibrutinib ist außerdem zur Behandlung erwachsener Patienten mit $\mathrm{CLL}$, die mindestens eine vorangehende Therapie erhalten haben indiziert. Ibrutinib ist zudem zur Behandlung von erwachsenen Patienten mit rezidiviertem oder refraktärem Mantelzell-Lymphom (MCL) zugelassen. Des Weiteren ist Ibrutinib bei erwachsenen Patienten mit Morbus Waldenström (MW) zugelassen. Die Zulassung bezieht sich auf die Behandlung von Patienten, die mindestens eine vorangehende Therapie erhalten haben, sowie auf die Erstlinienbehandlung von Patienten mit MW, die für eine Chemoimmuntherapie nicht geeignet sind.

Abb. 1. Nach 18 Monaten zeigten Patienten im Ibrutinib-Arm ein besseres PFS gegenüber Patienten unter Chlorambucil ( $90 \%$ vs. $52 \%)$. Das mediane PFS betrug unter Chlorambucil 18,9 Monate, unter Ibrutinib wurde es noch nicht erreicht (prim. Endpunkt, IRC Bewertung; $\mathrm{p}<0,001 ; \mathrm{HR} 0,16$ 95\%-KI: 0,09-0,28), modifiziert nach [2]

\begin{abstract}
** Die nachhaltige hämatologische Verbesserung wurde definiert als eine Steigerung hämatologischer Parameter die kontinuierlich für mindestens 56 Tage ohne Transfusion oder Wachstumsfaktoren anhielt. Dies wurde wie folgt gemessen: als Anstieg der Thrombozytenzah oder absoluten Neutrophilenzahl um mindestens 50\% des Ausgangswertes oder als Anstieg des Hämoglobinwertes von $\geq 2 \mathrm{~g} / \mathrm{dl}$ vom Ausgangswert; bei Patienten mit Zytopenie als Ausgangslage: als Anstieg des Hämoglobinwertes auf $\geq 11 \mathrm{~g} / \mathrm{dl}$, eine Thrombozytenzahl von mehr als $100000 / \mathrm{mm}^{3}$ oder eine absolute Neutrophilenzahl von $>1500 / \mathrm{mm}^{3}$.
\end{abstract}




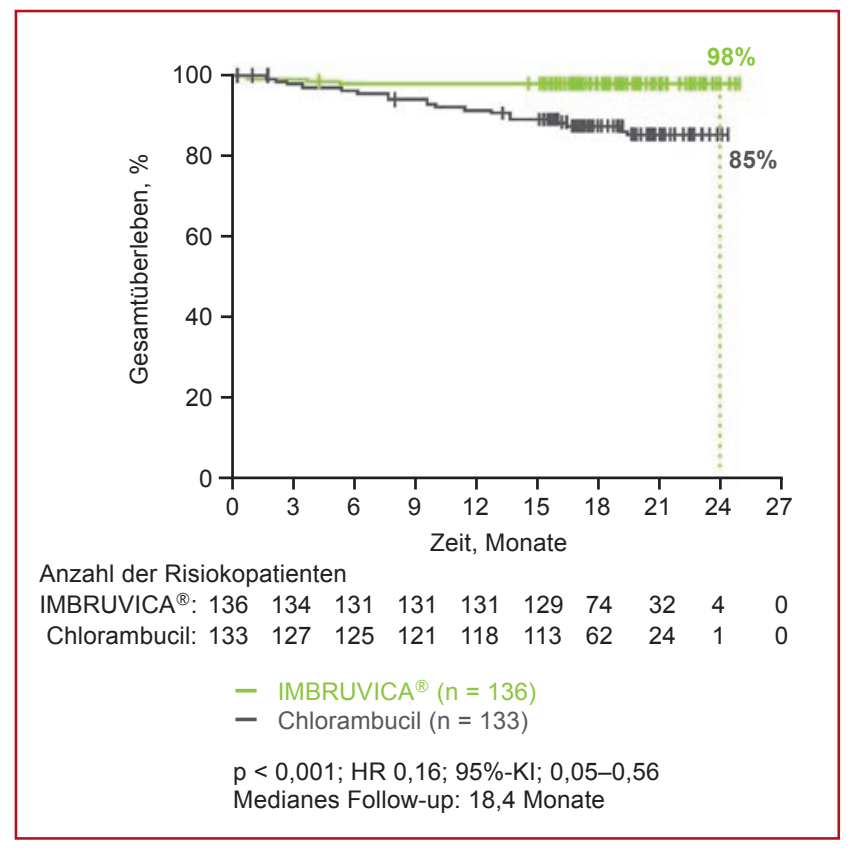

Abb. 2. Nach 2 Jahren waren signifikant mehr Patienten unter Ibrutinib am Leben als unter Chlorambucil. Nach 24 Monaten lebten noch $98 \%$ der Patienten im Ibrutinib-Arm versus $85 \%$ der Patienten im Chlorambucil-Arm ( $\mathrm{p}<0,001$; sekundärer Endpunkt), modifiziert nach [2].

Endpunkt) [2]. Diese Ergebnisse bestätigen die Daten einer vorangegangenen Phase-III-Studie (RESONATE) mit rez./ref. CLL-Patienten [4].

\section{Geringe Toxizität und hohe Verträglichkeit}

Der BTK-Inhibitor wurde auch in der Erstlinienbehandlung von älteren CLL-Patienten gut vertragen und bestätigte das bereits bekannte Sicherheitsprofil von Ibrutinib in der rezidivierten Situation [2, 5]. Trotz längerer Behandlungsdauer unter Ibrutinib als unter Chlorambucil (17,4 Monate vs. 7,1 Monate), war die Anzahl der nebenwirkungsbedingten Therapieabbrüche nur halb so hoch wie unter Chlorambucil ( $9 \%$ vs. 23\%) [2]. Während der Ibrutinib-Therapie wurden keine neuen Nebenwirkungen im Vergleich zu anderen Phase-III-Studien mit Ibrutinib beobachtet. In beiden Therapie-Armen war die Mehrheit der unerwünschten therapiebedingten Nebenwirkungen hauptsächlich vom Grad 1 und 2. $\mathrm{Zu}$ den häufigsten therapiebedingten Nebenwirkungen (aller Schweregrade), die bei $\geq 20 \%$ der Patienten im Ibrutinib-Arm auftraten, zählten Diarrhö (42\%), Fatigue (30\%), Übelkeit (22\%) und Husten (22\%). Im Chlorambucil-Arm gehörten Übelkeit (39\%), Fatigue (38\%), Neutropenien (23\%), Anämien (20\%) und Erbrechen (20\%) zu den häufigsten therapiebedingten Nebenwirkungen (aller Schweregrade), die bei $\geq 20 \%$ der Patienten beobachtet wurden [6]. Innerhalb des medianen Follow-ups von 18,4 Monaten gab es im Ibrutinib-Arm 3 Todesfälle, unter Chlorambucil waren es dagegen 17 Todesfälle [2].
In der Erstlinienbehandlung von therapienaiven CLL-Patienten zeigte sich Ibrutinib als hochwirksame Therapiealternative zur Chlorambucil-Chemotherapie mit guter Verträglichkeit.

\section{Ibrutinib: hohe Wirksamkeit auch bei rezidivier-} ten und refraktären Patienten

Seit Oktober 2014 ist Ibrutinib zur Behandlung von Patienten mit rez./ref. CLL zugelassen. Grundlage dafür war die vergleichende Phase-III-Studie RESONATE [5, 7]. In dieser Studie wurde die Therapie von rez./ref. CLL-Patienten mit Ibrutinib $(\mathrm{n}=195)$ mit der unter Ofatumumab $(n=196)$ gegenübergestellt $[5,7]$. Auch hier war Ibrutinib in den definierten Studienendpunkten dem Vergleichsarm signifikant überlegen. Patienten res 12-Monats-PFS als im Vergleichsarm (84\% vs. $18 \%$; p < 0,001; prim. Endpunkt) [7]. Nach einem medianen Follow-up von 16 Monaten lebten die Patienten unter Ofatumumab im Schnitt 8,1 Monate ohne Progression, unter Ibrutinib wurde der Median dagegen noch nicht erreicht [8]. Auch bezüglich anderer sekundärer Studienendpunkte wie OS und ORR profitierten Patienten deutlich mehr von Ibrutinib als von Ofatumumab. Nach 12 Monaten waren $90 \%$ der Patienten im Ibrutinib-Arm am Leben, im Vergleichsarm waren es noch $81 \%$ [5]. Zudem sprachen signifikant mehr Patienten auf die Behandlung mit Ibrutinib an $(90 \%)$, bei Ofatumumab war es nur ein Viertel der Patienten (25\%; $\mathrm{p}<0,0001)$ [8]. unter Ibrutinib zeigten ein signifikant besse-
Gemäß retrospektiver Subgruppenauswertungen wirkte Ibrutinib dabei unabhängig vom zytogenetischen Risikoprofil der Patienten. Das 12-Monats-PFS der Patienten mit 17p-Deletion unterschied sich nicht von dem der Patienten ohne Deletion [8]. Eine weitere Subgruppenauswertung ergab, dass der frühe Einsatz von Ibrutinib ab der Zweitlinie sich gegenüber späteren Therapielinien eindeutig positiv auf das PFS auswirkte [8]. Nach einem Beobachtungszeitraum von 12 Monaten blieben Patienten mit nur einer Vortherapie deutlich länger progressionsfrei als Patienten mit $>1$ Vortherapie ( $94 \%$ vs. $82 \% ; p=0,01)$ [8]. Die Knochenmarksfunktion der Patienten mit rez./ref. CLL verbesserte sich deutlich stärker während der Behandlung mit Ibrutinib als unter Ofatumumab. Die Anzahl der Thrombozyten $(\mathrm{p}<0,0001)$ und Neutrophilen $(\mathrm{p}=0,015)$ sowie der Hämoglobinspiegel $(p<0,0001)$ zeigten eine signifikante Verbesserung im Vergleich zu Ofatumumab [9].

Die Ergebnisse der 3-jährigen Langezeitbeobachtung der PCYC-1102/1103 (Phase-Ib/ II-Studie) [3] zeigten die anhaltend gute Verträglichkeit und Wirksamkeit von Ibrutinib bei 135 rez./ref. CLL-Patienten. Während dieser Beobachtung nahmen die Inzidenzen ausgewählter Nebenwirkungen $(\geq 20 \%)$ im Laufe der Therapiedauer ab [3].

Zusammenfassend zeigte Ibrutinib als Einzelsubstanz in allen Studien sowohl bei therapienaiven als auch bei rez./ref. Patienten eine der jeweiligen Vergleichssubstanz weit überlegene Wirksamkeit mit gleichzeitig guter Verträglichkeit. Die orale Gabe von Ibrutinib ist einfach und schnell anzuwenden.

\section{Literatur}

1 Aktuelle IMBRUVICA ${ }^{\circledR}$ Fachinformation.

2 Burger JA et al.: N Engl J Med 2015;373:2425-2437.

3 Byrd JC: Blood 2015;125:2497-2506.

4 Byrd JC et al.: N Engl J Med 2013;369:32-42.

5 Byrd JC et al.: N Engl J Med 2014;371:213-223

6 Burger JA et al.: N Engl J Med 2015;373:2425-2437, (Supplement).

7 Brown JR et al.: ASH 2014; Poster Presentation, abstr 3331.

8 Brown JR et al.: Blood 2014;124:abstr 3331.

9 Barrientos JC et al.: ASH 2014; Poster Presentation, abstr 4696.

Weitere Informationen bei

Janssen-Cilag GmbH

Tel. +492137955955

jancil@its.jnj.com

www.janssen-med.de 